\title{
Co-state Initialization for the Minimum-Time Low-Thrust Trajectory Optimization
}

\author{
Ehsan Taheri ${ }^{1, *}$, Nan I. Li ${ }^{2}$, Ilya Kolmanovsky ${ }^{3}$ \\ University of Michigan, Ann Arbor, Michigan 48109-2102
}

\begin{abstract}
This paper presents an approach for co-state initialization which is a critical step in solving minimum-time low-thrust trajectory optimization problems using indirect optimal control numerical methods. Indirect methods used in determining the optimal space trajectories typically result in two-point boundary-value problems and are solved by single- or multiple-shooting numerical methods. Accurate initialization of the co-state variables facilitates the numerical convergence of iterative boundary value problem solvers. In this paper, we propose a method which exploits the trajectory generated by the so-called pseudo-equinoctial and three-dimensional finite Fourier series shape-based methods to estimate the initial values of the co-states. The performance of the approach for two interplanetary rendezvous missions from Earth to Mars and from Earth to asteroid Dionysus is compared against three other approaches which, respectively, exploit random initialization of co-states, adjoint-control transformation and a standard genetic algorithm. The results indicate that by using our proposed approach the percent of the converged cases is higher for trajectories with higher number of revolutions while the computation time is much lower. These features are advantageous

\footnotetext{
*Corresponding author

Email addresses: etaheri@umich.edu (Ehsan Taheri ), nanli@umich.edu (Nan I. Li ), ilya@umich.edu (Ilya Kolmanovsky)

${ }^{1}$ Postdoctoral research fellow, Department of Aerospace Engineering, 1320 Beal Avenue, Ann Arbor, MI 48109, USA

${ }^{2} \mathrm{PhD}$ student, Department of Aerospace Engineering, 1320 Beal Avenue, Ann Arbor, MI 48109, USA.

${ }^{3}$ Full Professor, Department of Aerospace Engineering, 1320 Beal Avenue, Ann Arbor, MI 48109, USA.
} 
for broad trajectory search in the preliminary phase of mission designs.

Keywords: Trajectory optimization; Low-thrust; Shape-based;

Minimum-time; Indirect method

\section{Nomenclature}

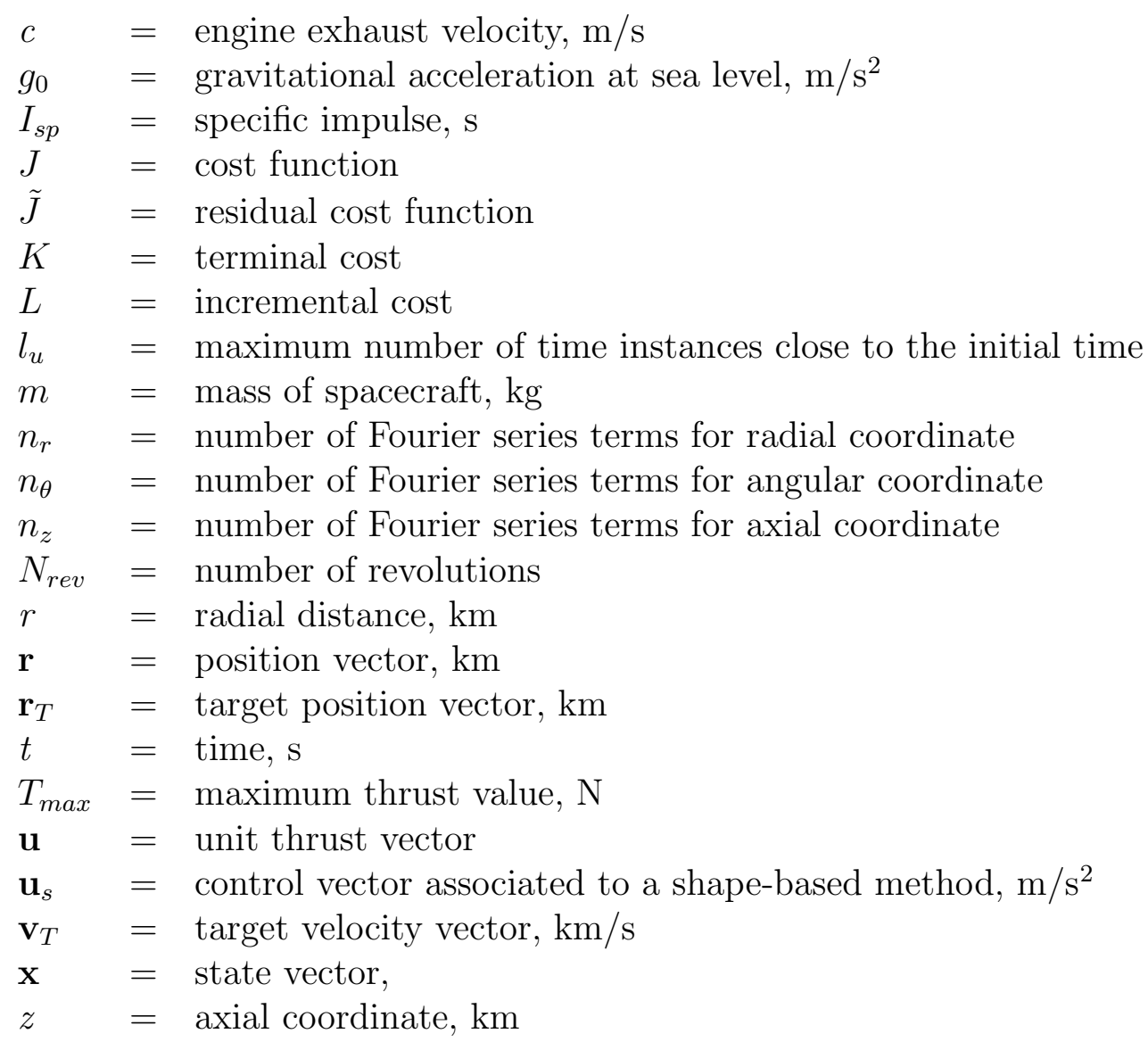




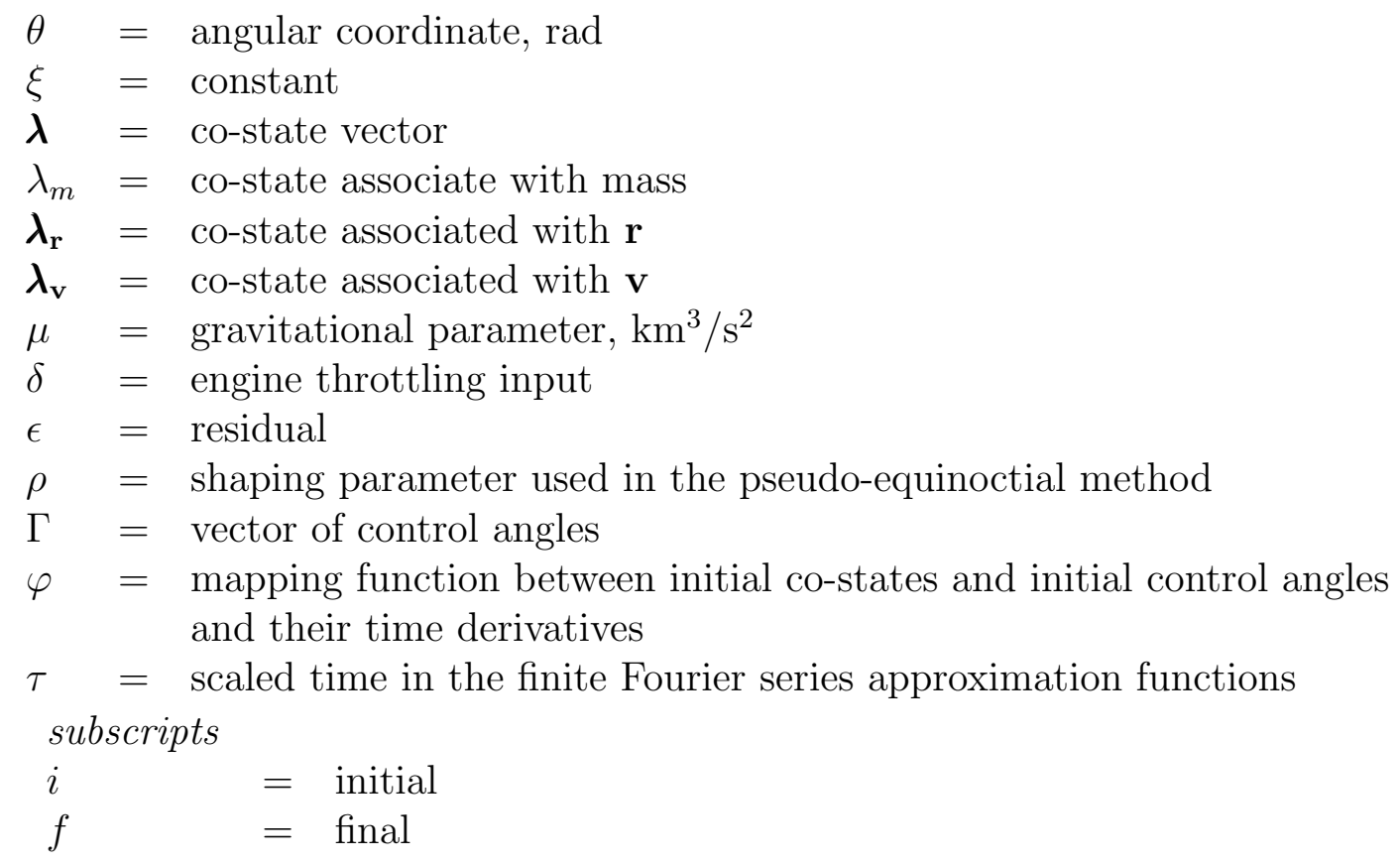

\section{Introduction}

The trajectory optimization problems can be generally solved by either indirect or direct techniques (Betts, 1998). The indirect approaches are based on analytically deriving the necessary conditions for optimality based on the Pontryagin's Maximum Principle (PMP) and solving the resulting twopoint boundary-value problem (TPBVP) numerically. Direct methods, on the other hand, exploit conversion of the optimal control problem into a nonlinear parameter optimization problem with various transcription schemes (e.g., Hermite cubic, cubic polynomial, and orthogonal function approximations) of either states or controls or both states and controls. The direct methods are further subdivided into two variants: simultaneous and sequential (Kamyar and Taheri, 2014). In the former, the transcription into a finite dimensional Non-Linear Programming (NLP) problem is achieved through discretization of both control and state variables, whereas in the latter, only control variables are discretized.

In the indirect method, the introduction of the so-called co-states (Lagrange multipliers) doubles the number of the differential equations that have to be propagated along the trajectory. Furthermore, the major difficulty in using the indirect techniques is the sensitivity of the solution to the 
unknown initial co-state values. As a consequence, the neighborhood of the true co-state values from which the solver converges to the optimal solution is often small (Betts, 1998). Moreover, the derivation of the Hamiltonian and first-order necessary conditions as well as the transversality conditions can be tedious for larger problems. At the same time, indirect techniques continue to be of interest for space applications in which the equations of motion (EoM) are relatively low order; when combined with proper initialization and homotopy procedures these methods are able to achieve fast convergence to a solution of the necessary conditions (Bertrand and Epenoy, 2002; Jiang et al., 2012; Taheri et al., 2016a).

Both of the aforementioned direct and indirect methods aim at a highfidelity solution but may be time consuming and not suitable for evaluating thousands of trajectories in the preliminary phase of the mission design. To overcome some of the aforementioned problems various heuristic and/or evolutionary techniques have been proposed (Vasile et al., 2010; Abdelkhalik and Gad, 2012; Englander et al., 2012; Chilan and Conway, 2013; Hennes and Izzo, 2015).

For the indirect methods, several techniques for estimating the co-states have been proposed. For instance, reference (Dixon and Bartholomew-Biggs, 1981) introduces an adjoint control transformation (ACT) for estimating the co-states of the TPBVP arising in low-thrust trajectory design. In ACT approach, the initial steering angles of the control vector and their associated derivatives are used to compute the estimate of initial co-state values (Kluever and Pierson, 1997; Ranieri and Ocampo, 2005; Senent et al., 2005; Russell, 2007). In another approach (Thorne and Hall, 1996), the co-states are determined analytically by simplifying the dynamics of the original problem. The simplified dynamics are obtained by ignoring the effect of the central body (setting gravitational parameter to zero) and assuming no fuel consumption $(\dot{m}=0)$. However, this approach is limited to low-thrust trajectories of less than one revolution. In (Yan and $\mathrm{Wu}, 1999$ ), it is demonstrated that approximate values of the initial adjoint variables can be obtained by solving equations in the neighborhood of the initial time in which the adjoint variables can be expressed by a first-order Taylor series expansion.

The low-thrust trajectories require not only much longer time of flight compared to classical ones, but also tend to perform many revolutions around the attracting body. Averaging technique is an efficient method to have a good initial guess for the many-revolution low-thrust trajectories (Geffroy and Epenoy, 1997; Fourcade et al., 2000). 
Shape-based (SB) methods, on the other hand, represent the state-of-theart methods for rapid generation of feasible low-thrust trajectories (Vasile et al., 2007; Wall and Conway, 2009; Novak and Vasile, 2011; Gondelach and Noomen, 2015; Taheri and Abdelkhalik, 2012b,a, 2015, 2016). The solution trajectory determined by SB methods satisfies the EoM, boundary conditions (BCs) and, depending on the technique used, the constraint on the thrust acceleration. The advantage of using the solution of SB methods as an initial guess for direct solvers is investigated in (Taheri and Abdelkhalik, 2015, 2016).

In this paper, we propose a specific method to utilize the trajectory from $\mathrm{SB}$ methods to derive estimates for initial values of the co-states for the indirect method. Some preliminary results of this method is investigated in (Taheri et al., 2016b). In this work, the performance of the algorithm is compared against several other techniques, and is shown to even outperform that of the ACT. In addition, a more difficult problem is considered and the utilization of two shape-based methods are investigated. It is shown that the proposed approach enhances the numerical percent of convergence of the iterative TPBVP solver while being computationally less intensive.

This paper is organized as follows. In section 2, EoMs are explained and PMP is applied for deriving the necessary optimality conditions in the minimum-time optimal control problem. Details of the procedure for estimating the co-states of the TPBVP are also described. In section 3, we use the Earth-to-Mars and Earth-to-Dionysus rendezvous problems to demonstrate the performance of our method and compare it to the performance of three other methods for initializing the co-states (based on the random initialization, adjoint-control transformation technique and based on a standard genetic algorithm) along with discussions of the results. Finally, conclusions are summarized in section 4 .

\section{Problem Formulation}

In this section, the governing EoM are derived and the minimum-time lowthrust trajectory optimization problem is formulated. Then, the procedure

for estimating the co-states is explained and the mathematical relations are derived. In addition, the details of the ACT approach are explained when the EoM are expressed in an inertial Cartesian coordinate system (CS). 


\subsection{Differential Equations and Formulation of the Minimum-Time Problem}

We consider an optimal control problem for a spacecraft affected by the gravitational acceleration of a central body and the acceleration of the onboard propulsion system. All other perturbations are neglected. The motion dynamics are expressed in the inertial Cartesian CS and the variation of mass is included. Defining the state vector as $\mathbf{x}=\left[\mathbf{r}^{T}, \mathbf{v}^{T}, m\right]^{T}$, the dynamics can be written as $\dot{\mathbf{x}}=f(\mathbf{x}(t), \mathbf{u}(t), \delta(t))$,

$$
\begin{aligned}
& \dot{\mathbf{r}}=\mathbf{v}, \\
& \dot{\mathbf{v}}=-\frac{\mu}{r^{3}} \mathbf{r}+\frac{T_{\max }}{m} \delta \mathbf{u}, \\
& \dot{m}=-\frac{T_{\max }}{c} \delta,
\end{aligned}
$$

where $\mathbf{r}$ is the position vector, $r$ is its Euclidean norm, $\mathbf{v}$ is the velocity vector, $\mu$ is the gravitational parameter of the central body, $m$ is the mass of the spacecraft, $T_{\max }$ is the maximum available thrust, $0 \leq \delta \leq 1$ is the engine throttling, $\mathbf{u}$ is the unit direction vector of the thrust and $c=I_{s p} g_{0}$ is the exhaust velocity. $I_{s p}$ and $g_{0}$ are the specific impulse and the gravitational acceleration at sea level, respectively. The method proposed in this paper is applied to the minimum-time trajectory optimization problem for which the Bolza form of the cost function is written as

$$
J=K\left(\mathbf{x}\left(t_{f}\right), t_{f}\right)+\int_{t_{i}}^{t_{f}} L(\mathbf{x}(t), \mathbf{u}(t), \delta(t)) d t,
$$

where the terminal cost and the incremental cost are $K=0$ and $L=1$, respectively. This particular case, in which the cost does not depend on the terminal states, is called Lagrange problem. The control inputs being optimized are $\mathbf{u}$ and $\delta$. We proceed by forming the Hamiltonian

$$
\begin{aligned}
H & =\xi L+\lambda^{T} f(\mathbf{x}(t), \mathbf{u}(t), \delta(t)) \\
& =\xi+\lambda_{r}^{T} \mathbf{v}+\lambda_{v}^{T}\left(-\frac{\mu}{r^{3}} \mathbf{r}+\frac{T_{\max }}{m} \delta \mathbf{u}\right)+\lambda_{m}\left(-\frac{T_{\max }}{c} \delta\right),
\end{aligned}
$$

where $\xi \in\{0,1\}$. The coefficient $\xi$ measures the influence of the cost function in the Hamiltonian and its detailed explanations are given in Section 2.2. The weak form of the PMP is used to characterize the optimal thrust vector,

$$
\mathbf{u}^{*} \in \arg \min _{\|\mathbf{u}\|=1} H
$$


which corresponds to minimizing the following term in the Hamiltonian

$$
\frac{T_{\max }}{m} \delta \boldsymbol{\lambda}_{v}^{T} \mathbf{u} \rightarrow \min .
$$

The Hamiltonian is minimized when

$$
\mathbf{u}^{*}=-\frac{\lambda_{v}}{\lambda_{v}}=\frac{\mathbf{P}}{p},
$$

where $\lambda_{v}=\left\|\boldsymbol{\lambda}_{v}\right\|, \mathbf{P}$ is defined as the primer vector (Russell, 2007) and $p=\|\mathbf{P}\|$. In other words, the optimal policy is to keep the thrust vector, always, aligned with the primer vector. Exploiting the weak form of PMP we can also characterize the optimal throttling magnitude input,

$$
\delta^{*} \in \arg \min _{0 \leq \delta \leq 1} H .
$$

From (5), to determine $\delta$ we need to minimize the term,

$$
-T_{\max }\left(\frac{\lambda_{v}}{m}+\frac{\lambda_{m}}{c}\right) \delta \rightarrow \min .
$$

The optimal value of $\delta$ depends on the sign of the term inside the parenthesis. Singular arcs exist for cases in which the term in the parenthesis is zero over a finite time interval but they rarely occur in space trajectories (Russell, 2007). Therefore, assuming there are no singular arcs in the solution,

$$
\begin{aligned}
& \frac{\lambda_{v}}{m}+\frac{\lambda_{m}}{c}>0 \rightarrow \delta=1, \\
& \frac{\lambda_{v}}{m}+\frac{\lambda_{m}}{c}<0 \rightarrow \delta=0 .
\end{aligned}
$$

We next derive the co-states (adjoint) differential equations through $\dot{\lambda}=$ $-\left[\frac{\partial H}{\partial \mathbf{x}}\right]^{T}$,

$$
\begin{aligned}
& \dot{\boldsymbol{\lambda}}_{r}=-\left[\frac{\partial H}{\partial \mathbf{r}}\right]^{T}=\frac{\mu}{r^{3}} \boldsymbol{\lambda}_{v}-\frac{3 \mu \mathbf{r} \cdot \boldsymbol{\lambda}_{v}}{r^{5}} \mathbf{r}, \\
& \dot{\boldsymbol{\lambda}}_{v}=-\left[\frac{\partial H}{\partial \mathbf{v}}\right]^{T}=-\boldsymbol{\lambda}_{r}, \\
& \dot{\lambda}_{m}=-\frac{\partial H}{\partial m}=-\frac{T_{\max }}{m^{2}} \delta \lambda_{v} .
\end{aligned}
$$


In this work, we consider a rendezvous maneuver in which the final position and velocity vectors have to be equal to the target values. Thus, the BCs can be written in the form of equality constraints,

$$
g\left(\mathbf{x}\left(t_{f}\right), t_{f}\right)=\left[\begin{array}{c}
\mathbf{r}\left(t_{f}\right)-\mathbf{r}_{T} \\
\mathbf{v}\left(t_{f}\right)-\mathbf{v}_{T}
\end{array}\right]=\mathbf{0}
$$

where $\mathbf{r}_{T}$ and $\mathbf{v}_{T}$ are the position and velocity vectors of the target. Finally, the transversality conditions can be written as

$$
\lambda\left(t_{f}\right)=\sum_{k=1}^{6} \alpha_{k}\left[\frac{\partial g_{k}\left(\mathbf{x}\left(t_{f}\right), t_{f}\right)}{\partial \mathbf{x}}\right]^{T},
$$

where

$$
\boldsymbol{\lambda}_{r}\left(t_{f}\right)=\left[\begin{array}{l}
\alpha_{1} \\
\alpha_{2} \\
\alpha_{3}
\end{array}\right], \quad \boldsymbol{\lambda}_{v}\left(t_{f}\right)=\left[\begin{array}{l}
\alpha_{4} \\
\alpha_{5} \\
\alpha_{6}
\end{array}\right], \quad \lambda_{m}\left(t_{f}\right)=0 .
$$

The right-hand side of the co-state differential equation associated with the mass state, Eq. (15), is always non-positive, $\dot{\lambda}_{m} \leq 0$, and from the transversality condition $\lambda_{m}\left(t_{f}\right)=0$ which means that $\lambda_{m}(t) \geq 0$. This indicates that the term in the parenthesis of Eq. (10) is always positive and, hence, Eq. (11) always holds, i.e.,

$$
\frac{\lambda_{v}}{m}+\frac{\lambda_{m}}{c}>0 \rightarrow \delta(t)=1
$$

Thus in the minimum-time problem of the type we consider the throttle is at its maximum value along the optimal trajectory, i.e., $\delta=1$, which is a well-known fact, see (Yue et al., 2009). In addition, we have a condition on the Hamiltonian because the final time, $t_{f}$, is free

$$
H\left(\mathbf{x}^{*}\left(t_{f}\right), \xi, \lambda^{*}\left(t_{f}\right), \mathbf{u}^{*}\left(t_{f}\right), \delta^{*}\left(t_{f}\right)\right)=0 .
$$

On the other hand, neither the state equations, cost functional, nor the terminal constraints depend on time explicitly which means that the Hamiltonian is a constant along the optimal trajectory. Hence

$$
H\left(\mathbf{x}^{*}(t), \xi, \lambda^{*}(t), \mathbf{u}^{*}(t), \delta^{*}(t)\right)=0 .
$$

Therefore, the state differential equations, Eqs. (1)-(3), the co-state differential equations, Eq. (13)-(15), the terminal constraints, Eqs. (16), the terminal 
condition of $\lambda_{m}\left(t_{f}\right)=0$ and the condition on the final value of the Hamiltonian, $H\left(t_{f}\right)=0$, constitute the TPBVP associated with the minimum-time problem.

Any solution scheme developed for solving this problem requires an initial guess for the unknown values of the initial co-states, $\boldsymbol{\lambda}=\left[\boldsymbol{\lambda}_{r}^{T}, \boldsymbol{\lambda}_{v}^{T}, \lambda_{m}\right]^{T}$ and final time, $t_{f}$. In a single-shooting scheme, the problem can be written as

$$
\boldsymbol{\psi}\left(\boldsymbol{\lambda}\left(t_{i}\right), t_{f}\right)=\left[\left[\mathbf{r}\left(t_{f}\right)-\mathbf{r}_{T}\right]^{T},\left[\mathbf{v}\left(t_{f}\right)-\mathbf{v}_{T}\right]^{T}, \lambda_{m}\left(t_{f}\right), H\left(t_{f}\right)\right]^{T}=\mathbf{0}
$$

where $t_{f}$ is also one of the design variables along with the vector of unknown initial co-states, $\lambda\left(t_{i}\right)$.

\subsection{Initial Co-states Estimation Procedure}

The equations and relations that are used for co-state estimation are taken from (Yan and $\mathrm{Wu}, 1999$ ) and presented here for the sake of clarity and completeness. Necessary modifications are made to be consistent with the adopted notations used throughout the paper and the equations are developed for the case in which the differential equations are written in an inertial Cartesian CS. For the sake of simplicity we have dropped the '*' sign from all of the equations. In the previous section, it was shown that the Hamiltonian is zero along the optimal solution, Eq. (21), hence, at any time instance, $t_{l}$ $\left(l=1, \cdots, l_{u}\right)$,

$$
H\left(t_{l}\right)=\xi+\lambda\left(t_{l}\right)^{T} f\left(\mathbf{x}\left(t_{l}\right), \mathbf{u}\left(t_{l}\right)\right)=0 .
$$

Rearranging the above equation it follows that

$$
\lambda\left(t_{l}\right)^{T} f\left(\mathbf{x}\left(t_{l}\right), \mathbf{u}\left(t_{l}\right)\right)=-\xi .
$$

In the neighborhood of the initial time instance, $t_{i}$, the co-states can be approximated by the first-order Taylor expansion of $\boldsymbol{\lambda}(t)$ at $t_{i}$ as,

$$
\lambda\left(t_{l}\right)=\lambda\left(t_{i}\right)+\dot{\lambda}\left(t_{i}\right)\left(t_{l}-t_{i}\right) .
$$

From Eq. (24) and $\dot{\boldsymbol{\lambda}}\left(t_{i}\right)=-\left[\left.\frac{\partial H}{\partial \mathbf{x}}\right|_{t_{i}}\right]^{T}$, it follows that

$$
\left[\boldsymbol{\lambda}\left(t_{i}\right)-\left[\left.\frac{\partial H}{\partial \mathbf{x}}\right|_{t_{i}}\right]^{T}\left(t_{l}-t_{i}\right)\right]^{T} f\left(\mathbf{x}\left(t_{l}\right), \mathbf{u}\left(t_{l}\right)\right)=-\xi .
$$


By substituting for the state and co-states differential equations we obtain, from Eqs. (1)-(3), (13)-(15)

$$
\begin{gathered}
{\left[\begin{array}{c}
\boldsymbol{\lambda}_{r}\left(t_{i}\right)+\left[\frac{\mu}{r\left(t_{i}\right)^{3}} \boldsymbol{\lambda}_{v}\left(t_{i}\right)-\frac{3 \mu \mathbf{r}\left(t_{i}\right) \boldsymbol{\lambda}_{v}\left(t_{i}\right)}{r\left(t_{i}\right)^{5}} \mathbf{r}\left(t_{i}\right)\right] \\
\boldsymbol{\lambda}_{v}\left(t_{i}\right)-\boldsymbol{\lambda}_{r}\left(t_{i}\right)\left(t_{l}-t_{i}\right) \\
\lambda_{m}\left(t_{i}\right)-\frac{T_{\max }}{m\left(t_{i}\right)^{2}} \lambda_{v}\left(t_{i}\right)\left(t_{l}-t_{i}\right)
\end{array}\right]} \\
{\left[\begin{array}{c}
\mathrm{v}\left(t_{l}\right) \\
-\frac{\mu}{r\left(t_{l}\right)^{3}} \mathbf{r}\left(t_{l}\right)+\frac{T_{\max }}{m\left(t_{l}\right)} \mathbf{u}\left(t_{l}\right) \\
-\frac{T_{\max }}{c}
\end{array}\right]=-\xi .}
\end{gathered}
$$

Equation (27) has several unknowns which are the 7 initial co-states $\boldsymbol{\lambda}_{r}\left(t_{i}\right) \in \mathbb{R}^{3}, \boldsymbol{\lambda}_{v}\left(t_{i}\right) \in \mathbb{R}^{3}$ and $\lambda_{m}\left(t_{i}\right) \in \mathbb{R}^{1}$. In principle, it is sufficient to select 7 different time instances $t_{l}$ near the initial time $t_{i}$, so that we have equal number of equations and unknowns and can solve for $\lambda\left(t_{i}\right) \in \mathbb{R}^{7}$. But Eq. (27) is nonlinear and the solution to the set of 7 equations is not unique. We want the specific solution for $\boldsymbol{\lambda}\left(t_{i}\right)$ that corresponds to the trajectory of the SB method. In principle, we can construct an over-determined system - in which the number of equations exceeds the number of unknowns - by picking more than 7 different time instances $t_{l}$ close to $t_{i}$. A least-square residual problem can be formed for which the cost function is defined as

$$
\tilde{J}=\sum_{l=1}^{l_{u}} \epsilon_{l}^{2},
$$

where $\epsilon_{l}$ is the error of $l$-th equation, and $l_{u} \geq 7$. In this way, the minimum error solution of initial co-states $\boldsymbol{\lambda}\left(t_{i}\right)$, which converges to the trajectory of the SB method, can be obtained numerically. The solution is no longer unique which is in fact advantageous since it provides a pool of initial guesses which is favourable in the early phases of any low-thrust mission design. In Eq. (27), $\xi \in\{0,1\}$.

According to the general form of PMP, in the construction of the Lagrangian (as well as in the construction of the Hamiltonian), one should consider both the cases of $\xi=0$ and $\xi$ being a positive number. In some optimal control problem formulations, one sets $\xi$ to be a positive number by default by implicitly assuming some regularity condition (or constraint qualification condition) holds. In fact, this assumption usually works well. The case of $\xi=0$, informally speaking, implies a solution candidate that is 
entirely determined by the constraints, without really taking the cost into account.

The trajectories generated by SB methods only satisfy the constraints (including the initial condition and terminal condition, etc.), and do not really minimize the time of flight. Thus, it is not surprising that by dropping the term of cost in the Hamiltonian $(\xi=0)$, better estimates for the values of the co-estates are obtained. Through numerous computational experiments, we have established that by setting $\xi=0$, better results are found.

\subsection{ACT for Minimum-Time problem}

The underlying idea behind the ACT approach is to construct a mapping between the physical control variables (and their time derivatives) and the initial values of the co-states. It is based on the strong form of the PMP as

$$
\frac{\partial H}{\partial u_{i}}=0
$$

where $u_{i}$ is the $i$ th component of the control and

$$
\frac{\mathrm{d}^{p}}{\mathrm{dt}^{p}} \frac{\partial H}{\partial u_{i}}=0
$$

where $p$ is the order of the time derivative. Equation (29) holds along the optimal trajectory for the cases in which the control, $u$ is unconstrained. For the constrained control, and for the minimum-time problem in which $\delta(t)=1$, the weak form of the PMP is used to characterize the optimal direction of the control vector as

$$
\mathbf{u}^{*} \in \arg \min _{\|\mathbf{u}\|=1} H
$$

where $\mathbf{u}=\left[u_{1}, u_{2}, u_{3}\right]^{T}$. However, if we define the components of the direction vector as, $\mathbf{u}=[\cos \alpha \cos \beta, \sin \alpha \cos \beta, \sin \beta]^{T}$, the equality constraint of $\|\mathbf{u}\|=1$ is automatically satisfied. The new control variables, thus, become $\Gamma=[\alpha, \beta]^{T}$. Therefore, Eqs. (29) and (30) become

$$
\begin{gathered}
\frac{\partial H}{\partial \Gamma_{j}}=0, j=1,2 . \\
\frac{\mathrm{d}^{p}}{\mathrm{dt}^{p}} \frac{\partial H}{\partial \Gamma_{j}}=0, j=1,2 .
\end{gathered}
$$


The new Hamiltonian can be written as

$$
\begin{aligned}
\tilde{H} & =\xi L+\boldsymbol{\lambda}^{T} f(\mathbf{x}(t), \mathbf{u}(t))=\xi+\boldsymbol{\lambda}_{r}^{T} \mathbf{v}+\boldsymbol{\lambda}_{v}^{T}\left(-\frac{\mu}{r^{3}} \mathbf{r}+\frac{T_{\max }}{m} \mathbf{u}(\Gamma)\right)+\lambda_{m}\left(-\frac{T_{\max }}{c}\right) \\
& =C+\frac{T_{\max }}{m} \boldsymbol{\lambda}_{v}^{T} \mathbf{u}(\Gamma) \\
& =C+\frac{T_{\max }}{m}\left(\lambda_{v 1} \cos \alpha \cos \beta+\lambda_{v 2} \sin \alpha \cos \beta+\lambda_{v 3} \sin \beta\right)
\end{aligned}
$$

where $C$ is the sum of all terms without $\mathbf{u}$, and the term $\frac{T_{\max }}{m}$ is always positive. Both of these terms do not affect Eqs. (32) and (33) and are dropped from the Hamiltonian for the sake of deriving simpler expressions. The new Hamiltonian takes the following form

$$
\tilde{H}=\lambda_{v 1} \cos \alpha \cos \beta+\lambda_{v 2} \sin \alpha \cos \beta+\lambda_{v 3} \sin \beta .
$$

From Eq. (32) we can write

$$
\begin{gathered}
\frac{\partial \tilde{H}}{\partial \alpha}=-\lambda_{v 1} \sin \alpha \cos \beta+\lambda_{v 2} \cos \alpha \cos \beta=0, \\
\frac{\partial \tilde{H}}{\partial \beta}=-\lambda_{v 1} \cos \alpha \sin \beta-\lambda_{v 2} \sin \alpha \sin \beta+\lambda_{v 3} \cos \beta=0 .
\end{gathered}
$$

From Eq. (33) we can also write

$$
\frac{\mathrm{d}}{\mathrm{dt}} \frac{\partial \tilde{H}}{\partial \alpha}=0, \frac{\mathrm{d}}{\mathrm{dt}} \frac{\partial \tilde{H}}{\partial \beta}=0, \frac{\mathrm{d}^{2}}{\mathrm{dt}^{2}} \frac{\partial \tilde{H}}{\partial \alpha}=0, \frac{\mathrm{d}^{2}}{\mathrm{dt}^{2}} \frac{\partial \tilde{H}}{\partial \beta}=0 .
$$

The derivatives of the co-state vectors, $\dot{\boldsymbol{\lambda}}_{v}$ or $\dot{\boldsymbol{\lambda}}_{r}$, can be expanded in these expressions using Eqs. (13)-(15). Ultimately, a set of functions (which are linear in co-states) are obtained as $\left(\boldsymbol{\lambda}_{r}, \boldsymbol{\lambda}_{v}\right)=\varphi(\mathbf{r}, \alpha, \dot{\alpha}, \ddot{\alpha}, \beta, \dot{\beta}, \ddot{\beta})$. The relations in Eq. (37) are derived using symbolic toolbox of MATLAB. In addition, by inspecting Eqs. (35)-(37), the following linear function can be written

$$
A(\mathbf{r}, \alpha, \dot{\alpha}, \ddot{\alpha}, \beta, \dot{\beta}, \ddot{\beta})\left[\begin{array}{c}
\boldsymbol{\lambda}_{r} \\
\lambda_{v}
\end{array}\right]=\mathbf{0} .
$$

For any solution $\left[\left(\boldsymbol{\lambda}_{r}^{0}\right)^{T},\left(\boldsymbol{\lambda}_{v}^{0}\right)^{T}\right]^{T}$ of the following relation

$$
A\left[\begin{array}{l}
\lambda_{r}^{0} \\
\lambda_{v}^{0}
\end{array}\right]=\mathbf{0}
$$


$c\left[\left(\boldsymbol{\lambda}_{r}^{0}\right)^{T},\left(\boldsymbol{\lambda}_{v}^{0}\right)^{T}\right]^{T}$ is also a solution. The fact that any scaling of a solution of $\left(\boldsymbol{\lambda}_{r}, \boldsymbol{\lambda}_{v}\right)$ will be a valid solution for $\varphi$ is consistent with the fact that for the case of $\xi=0$, any scaling of a solution of the co-state vector $\lambda$ is a valid solution for $\boldsymbol{\psi}$ (See Eq. (22)).

In other words, Eqs. (35), (36) and (37) are linearly dependent and have one redundancy. We are free to choose one element of the co-state vector, $\left(\boldsymbol{\lambda}_{r}, \boldsymbol{\lambda}_{v}\right)$, as a constant and solve for the other 5 unknowns. We assumed $\lambda_{r 1}$ to be constant. The mapping, thus, becomes

$$
\left(\lambda_{r 2}, \lambda_{r 3}, \lambda_{v 1}, \lambda_{v 2}, \lambda_{v 3}\right)=\varphi\left(\lambda_{r 1}, \mathbf{r}, \alpha, \dot{\alpha}, \ddot{\alpha}, \beta, \dot{\beta}, \ddot{\beta}\right)
$$

Since we are interested in finding the initial values of the co-states, at the time instance, $t_{i}$, the mapping is written as

$$
\begin{array}{r}
\left(\lambda_{r 2}\left(t_{i}\right), \lambda_{r 3}\left(t_{i}\right), \lambda_{v 1}\left(t_{i}\right), \lambda_{v 2}\left(t_{i}\right), \lambda_{v 3}\left(t_{i}\right)\right)= \\
\varphi\left(\lambda_{r 1}\left(t_{i}\right), \mathbf{r}\left(t_{i}\right), \alpha\left(t_{i}\right), \dot{\alpha}\left(t_{i}\right), \ddot{\alpha}\left(t_{i}\right), \beta\left(t_{i}\right), \dot{\beta}\left(t_{i}\right), \ddot{\beta}\left(t_{i}\right)\right) .
\end{array}
$$

We want to utilize a trajectory of the SB method to initialize $\left(\alpha\left(t_{i}\right), \dot{\alpha}\left(t_{i}\right), \ddot{\alpha}\left(t_{i}\right), \beta\left(t_{i}\right), \dot{\beta}\left(t_{i}\right), \ddot{\beta}\left(t_{i}\right)\right)$. Since the control trajectory is prescribed by SB method at every time i.e., $\mathbf{u}_{s}(t)=\left[u_{s, 1}(t), u_{s, 2}(t), u_{s, 3}(t)\right]^{T}$ we can solve for $\alpha(t), \beta(t)$ through the following relations

$$
\begin{aligned}
\alpha(t) & =\operatorname{atan} 2\left(u_{s, 2}(t), u_{s, 1}(t)\right), \\
\beta(t) & =\operatorname{asin}\left(u_{s, 3}(t)\right) .
\end{aligned}
$$

We can also calculate numerically the values of the time derivatives of the control angles, i.e., $(\dot{\alpha}(t), \ddot{\alpha}(t), \dot{\beta}(t), \ddot{\beta}(t))$, at the initial time instant $t_{i}$. The initial position vector, $\mathbf{r}\left(t_{i}\right)$, is also known. By setting a value for $\lambda_{r 1}\left(t_{i}\right)$, we can get values for all $\left(\boldsymbol{\lambda}_{r}\left(t_{i}\right), \boldsymbol{\lambda}_{v}\left(t_{i}\right)\right)$. In the numerical simulations, we set $\lambda_{r 1}\left(t_{i}\right)=0.1$. This value matters because we still need to initialize $\lambda_{m}\left(t_{i}\right)$, and their ratio matters, but by numerical simulations we found that $\lambda_{r 1}\left(t_{i}\right)=0.1$ generates acceptable results. We initialize $\lambda_{m}\left(t_{i}\right)$ to a random value in the interval $[0,1]$.

\section{Results}

In this section, we assess the performance of our proposed method in improving the convergence rate by applying it to two interplanetary minimumtime rendezvous problems from Earth to Mars and from Earth to asteroid 
Dionysus. As was mentioned in Section 2, the first step in the solution procedure is to rapidly compute a feasible trajectory using one of the existing SB methods. In this paper, we considered two of the SB methods that are capable of generating three-dimensional (3D) trajectories, specifically, the pseudo-equinoctial (PE) (Pascale and Vasile, 2006) and 3D finite Fourier series (3D FFS) (Taheri and Abdelkhalik, 2016) SB methods. These SB methods are used to determine approximate trajectories of $\mathbf{r}$ and $\mathbf{v}$, in the inertial Cartesian CS, that are going to be exploited for the initial co-state estimation.

Note that the main motivation behind developing SB methods is to rapidly generate feasible solutions with the fewest number of parameters. An important feature of the considered SB methods is that they not only satisfy the BCs and EoM but are also capable of handling constraints on thrust acceleration, albeit by using different approaches.

For the PE SB method, there are ten free constants for pseudo-elements that satisfy the BCs and the remaining free parameters, $\rho_{i}(i=1, \cdots, 3)$, are used to properly shape pseudo-elements in order to meet the requirements and constraints on the thrust acceleration profile (Pascale and Vasile, 2006). However, in this work, we did not impose thrust acceleration constraints to speed up the algorithm and instead limited the range of the $\rho_{i}$ coefficients such that the resulting thrust accelerations remain within acceptable limits. For the 3D FFS method (Taheri and Abdelkhalik, 2016), the shape of the states (radial, angular and axial coordinates in the cylindrical CS) are approximated assuming a finite number of terms in the Fourier series,

$$
\begin{aligned}
& r(\tau)=\frac{a_{0}}{2}+\sum_{n=1}^{n_{r}}\left\{a_{n} \cos (n \pi \tau)+b_{n} \sin (n \pi \tau)\right\}, \\
& \theta(\tau)=\frac{c_{0}}{2}+\sum_{n=1}^{n_{\theta}}\left\{c_{n} \cos (n \pi \tau)+d_{n} \sin (n \pi \tau)\right\}, \\
& z(\tau)=\frac{e_{0}}{2}+\sum_{n=1}^{n_{z}}\left\{e_{n} \cos (n \pi \tau)+f_{n} \sin (n \pi \tau)\right\},
\end{aligned}
$$

where $0 \leq \tau=\frac{t}{T O F} \leq 1$ is the scaled time, TOF is the corresponding total time of flight. $n_{r}, n_{\theta}$ and $n_{z}$ are the number of Fourier terms for each state. The free coefficients of these Fourier approximations become the design parameters. For the 3D FFS SB method, we have the potential to include a 
constraint on the maximum level of the thrust acceleration or even consider an unconstrained problem whichever suits the application.

However, for a general rendezvous problem using the above two mentioned SB methods, the number of revolutions, $N_{\text {rev }}$, and the total time of flight, $T O F$, are two important parameters that should be known a priori either by using simplifying assumptions or established through a trial and error procedure. In fact, there should be a meaningful relationship between these two parameters, consistent with the differential equations, that increases the convergence rate. For instance, in the case that the thrust is aligned in the direction of the velocity vector (and assuming that the trajectory start on a circular orbit and is determined by the planar two-body dynamics), there exist relations between the time of flight and the number of revolutions (Perkins, 1959). These approximations can be used for a rough estimation of the number of revolutions. In addition, in order to speed up the solution procedure, a compiled code based on a modified version of MATLAB ode 45 function is used, which is based on a variable step explicit Ruge-Kutta formula, with absolute and relative tolerances set to $1.0 \times 10^{-6}$. The modified version is based on the MATLAB ode 45 code except that the intermediate variables are removed and no longer stored since the final value of states and co-states are of our interest and it provides a significant speed-up.

The performance of the proposed approach that relies on the trajectory from SB methods for co-state initialization is further compared against the performance of three other approaches i.e., random initialization, a standard genetic algorithm and ACT. In all of the cases, canonical units are used, such that one distance unit (DU) is equal to one astronomical unit (AU) and $2 \pi$ Time Unit (TU) is equal to 1 year. In addition, in the numerical simulations, the gravitational parameter of the Sun is set to $\mu=$ $1.32712440018 \times 10^{11} \mathrm{~km}^{3} / \mathrm{s}^{2}$ and $g_{0}=9.80665 \mathrm{~m} / \mathrm{s}^{2}$. MATLAB fmincon and $g a$ functions are used as the solvers, respectively. All of the numerical computations have been performed on an Intel Xeon 3.5 GHz PC with Windows 7 and 16GB of RAM. The reported computation times are calculated by using the MATLAB tic-toc command.

\subsection{Earth-to-Mars Problem}

In the simulations, the specific impulse is $I_{s p}=2000 s$ and the initial mass of the spacecraft is $m_{0}=1000 \mathrm{~kg}$. The BCs are taken from (Lantoine and Russell, 2012) where the position and velocity of Earth and 
Mars are given by

$$
\begin{aligned}
\mathbf{r}_{i} & =[-140699693,-51614428,980]^{T}(\mathrm{~km}) \\
\mathbf{v}_{i} & =\left[9.774596,-28.07828,4.337725 \times 10^{-4}\right]^{T}(\mathrm{~km} / \mathrm{s}), \\
\mathbf{r}_{T} & =[-172682023,176959469,7948912]^{T}(\mathrm{~km}), \\
\mathbf{v}_{T} & =\left[-16.427384,-14.860506,9.21486 \times 10^{-2}\right]^{T}(\mathrm{~km} / \mathrm{s}) .
\end{aligned}
$$

The departure from Earth occurs on April 10th, 2007 and the final Mars conditions correspond to a time of flight (TOF) of 348.795 days. However, this TOF corresponds to zero revolution $\left(N_{\text {rev }}=0\right)$ around Sun. Nevertheless, for the cases with a greater number of revolutions, we will use the same position and velocity vectors of $\operatorname{Mars}\left(\mathbf{r}_{T}, \mathbf{v}_{T}\right)$ as the terminal constraints for the rendezvous problem.

The eccentricity and inclination values of Mars are 0.093 and $1.85^{\circ}$ (with respect to the ecliptic), respectively, which makes it a relatively easy target. In order to consider harder problems, various low levels of thrust are considered such that the number of revolutions increases significantly. For each case, we first use a SB method to create a trajectory, then use this trajectory to estimate initial values of the co-states. MATLAB fmincon is used to minimize the function defined in Eq. (28) with 30 time instances i.e., $l_{u}=30$. Then the estimated values of the co-states are fed into the indirect solver to find local minima. Note that the BCs are the same for each thrust level.

For the random initialization, we used rand command in MATLAB whose returned value is a pseudorandom number in the range of $[0,1]$ with a uniform distribution. Finally, convergence performance of the indirect approach for various initialization techniques is presented and compared. Table 1 provides the details of the performance of the solver for three different thrust levels. As the thrust values become smaller and the number of revolutions increases, the benefit of using our proposed method becomes noticeable. Indeed, the number of required iterations and function evaluations gets smaller than when using random initialization. For different values of the number of revolutions, $N_{\text {rev }}$, both of the initialization procedures based on the solution of PE and 3D FFS SB methods require fewer number of iterations and function evaluations. It is known that for problems with longer TOF and higher number of revolutions single-shooting solution schemes encounter difficulty as the sensitivity of the problem with respect to the initial co-states increases. The last column in Table 1 provides the minimum TOF for each thrust level, and as it is expected, the time of flight increases for lower thrust values. Figures 1, 2 and 3 depict the minimum-time trajectories of the three 
Table 1: Comparison between the convergence performance for three different thrust levels for the given BCs.

\begin{tabular}{|c|c|c|c|c|c|c|c|}
\hline $\begin{array}{c}\text { Tmax } \\
(\mathrm{N})\end{array}$ & $N_{\text {rev }}$ & $\begin{array}{l}\text { Iter. \& Func-count } \\
\text { using PE }\end{array}$ & $\begin{array}{r}\text { Iter. \& } \\
\text { using }\end{array}$ & $\begin{array}{l}\text { nc-count } \\
\text { D FFS }\end{array}$ & $\begin{array}{l}\text { Iter. \& F } \\
\text { using ran }\end{array}$ & $\begin{array}{l}\text { unc-cc } \\
\text { dom g }\end{array}$ & $\begin{array}{l}\text { TOF } \\
\text { (days) }\end{array}$ \\
\hline 0.5 & 0 & $\begin{array}{ll}20 & 198 \\
\end{array}$ & 14 & 140 & 26 & 255 & 307.58 \\
\hline 0.05 & 2 & 91 & 42 & 405 & 48 & 469 & 1320.56 \\
\hline 0.015 & 10 & 513 & 29 & 280 & 82 & 793 & 4740.038 \\
\hline
\end{tabular}

cases in the inertial Cartesian CS, respectively. The small scale of the $z$ coordinate is kept to emphasize the three-dimensional transfer, although the inclination change is small.

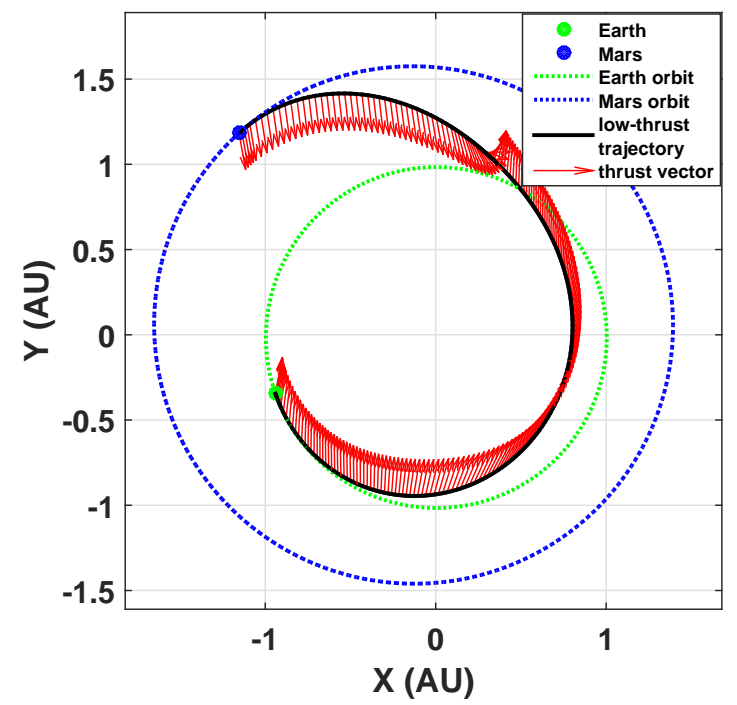

Figure 1: Minimum-time trajectory for the given BCs with $N_{\text {rev }}=0$.

Figure 4 shows the evolution of radius during the trajectory for the three thrust levels. It is interesting to note that for the case with two revolutions, the radius is always greater than the radius of the initial departure orbit. It is worth mentioning that the variation of orbital parameters, and in this case the radius value, mainly depends on the combination of several factors such as the BCs, time of flight and thrust level.

We further considered a case requiring a very high number of revolutions by considering a thrust value of $T_{\max }=0.0045 \mathrm{~N}$ which results in a trajectory with 30 revolutions. Table 2 compares the convergence rate of three 


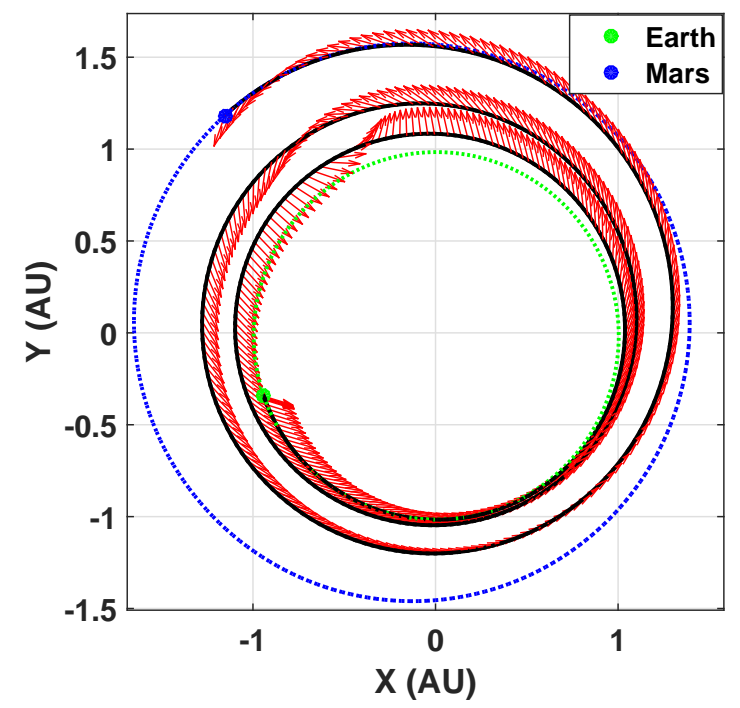

Figure 2: Minimum-time trajectory for the given BCs with $N_{\text {rev }}=2$.

approaches for 100 executions.

First, we considered the random initialization technique for the co-states and ran the numerical simulation of the shooting problem, Eq. (22), 100 times. At this high number of revolutions, the dependence of convergence on the quality of initial guess becomes so sensitive that only 15 times out of 100 random initial guesses converge.

Unlike the random initialization, the convergence rate with the proposed $\mathrm{PE}$ approach is $76 \%$ which is the best among alternatives covered and exceeds that of $46 \%$ when PE is used with the ACT technique (PE-ACT). For the $\mathrm{PE}$ initialization, the $24 \%$ non-convergence cases are mostly associated with the fact when we minimize Eq. (28), we use fmincon with a random initial guess.

Although we try to locate the solution corresponding to the trajectory from SB method by adding the number of equations, it is still possible to converge to some local minima other than the true one, but with a quite lower likelihood. Figure 5 shows the trajectory with 30 revolutions in the inertial Cartesian CS. The TOF for the plotted trajectory is 14990.56 days.

In addition, we compare the results of our proposed technique with a standard Genetic Algorithm (GA) which is a stochastic optimization algorithm (Goldberg, 1989). When dealing with complicated optimization problems 


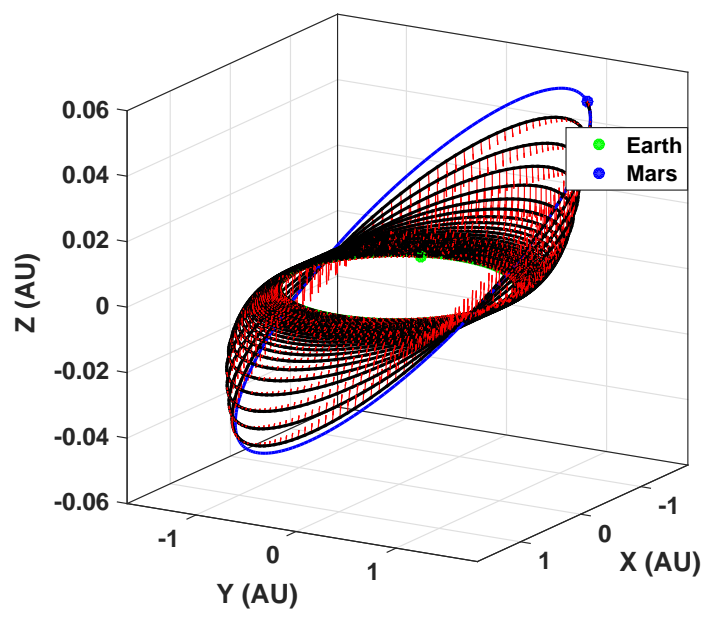

Figure 3: Minimum-time trajectory for the given BCs with $N_{\text {rev }}=10$.

with relatively small region of convergence, a hybrid algorithm 'GA + Fmincon= GAFM' is deemed to achieve a relatively good rate of convergence by creating a pool of sufficiently large population and enough number of generations.

Table 2: Comparison between the convergence rate of the proposed approach and random initialization for the given BCs for $T_{\max }=0.0045 \mathrm{~N}$ and with $N_{r e v}=30$

\begin{tabular}{|c|c|c|c|c|}
\hline $\begin{array}{c}\text { Rate of conv. } \\
\text { using PE }\end{array}$ & $\begin{array}{c}\text { Rate of conv. } \\
\text { using PE-ACT }\end{array}$ & $\begin{array}{c}\text { Rate of conv. } \\
\text { using 3D FFS }\end{array}$ & $\begin{array}{c}\text { Rate of conv. } \\
\text { using 3D FFS-ACT }\end{array}$ & $\begin{array}{c}\text { Rate of conv. } \\
\text { using rand. guesses }\end{array}$ \\
\hline $76 \%$ & $46 \%$ & $53 \%$ & $46 \%$ & $15 \%$ \\
\hline
\end{tabular}

By GA we first find a relatively good point, which is very likely to be located in the neighborhood of a local minimum. Then, we feed this point to fmincon as an initial guess to enhance the convergence to a local minimum more accurately.

The advantage of using stochastic/evolutionary algorithms is that 1) they do not need an initial guess and 2) the chances that the solver is trapped in a local minima is lower compared with the gradient-based methods. However, the drawback of using stochastic approaches, like GA, is that they usually require a large number of function evaluations and, thus, require long computational time. In the case that the population is not large enough, the rate of convergence may decline considerably. The problem becomes worse when 


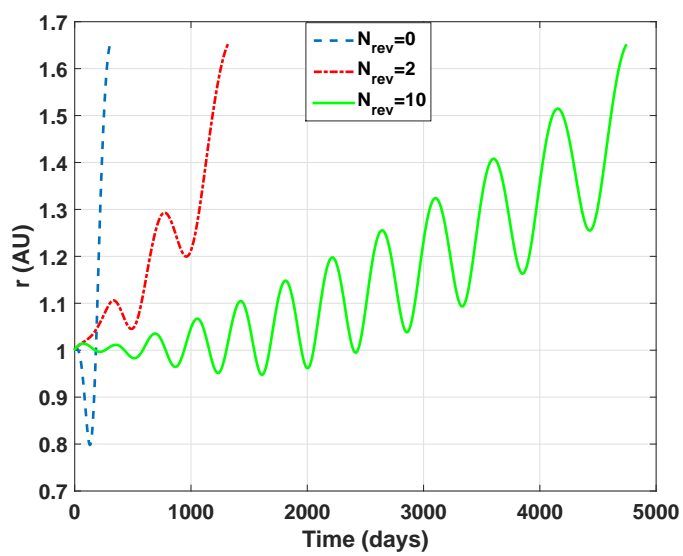

Figure 4: Radius value v.s. time for the three thrust levels of the minimum-time problem for the given BCs.

the dimension of the vector of unknown parameters is large.

As for the 7 dimensional parameter space of our case, even with parallel computing, the computational load is still quite high. For instance, we considered the previous problem with 30 revolutions as the test case and compared the performance of the algorithms for various populations. Table 3 compares the performance of the algorithms.

Table 3: Convergence rate and computational time comparison between GA and SB Initialization

\begin{tabular}{|c|c|c|c|c|c|}
\hline Initialization method & $\begin{array}{c}\text { Rate of } \\
\text { conv. }\end{array}$ & $\begin{array}{c}\text { Ave. comp. } \\
\text { time (sec) }\end{array}$ & $\begin{array}{c}\text { No. of } \\
\text { cores }\end{array}$ & Population & Generation \\
\hline PE & $76 \%$ & 27 & - & - & - \\
\hline 3D FFS & $53 \%$ & 15 & - & - & - \\
\hline PE-ACT & $46 \%$ & 10 & - & - & - \\
\hline 3D FFS-ACT & $46 \%$ & 8 & - & - & - \\
\hline GAFM & $64 \%$ & 80 & 4 & 5000 & 5 \\
\hline GAFM & $48 \%$ & 40 & 4 & 2000 & 5 \\
\hline
\end{tabular}

We used parallel computing capability of MATLAB and used the maximum number of available cores. In addition, the stochastic algorithms had to be executed several times to make sure that they converge to the optimal solution. Table 3 reports the results of GA with two different population sizes.

The rate of convergence with the $\mathrm{SB}$ initialization is better than with 


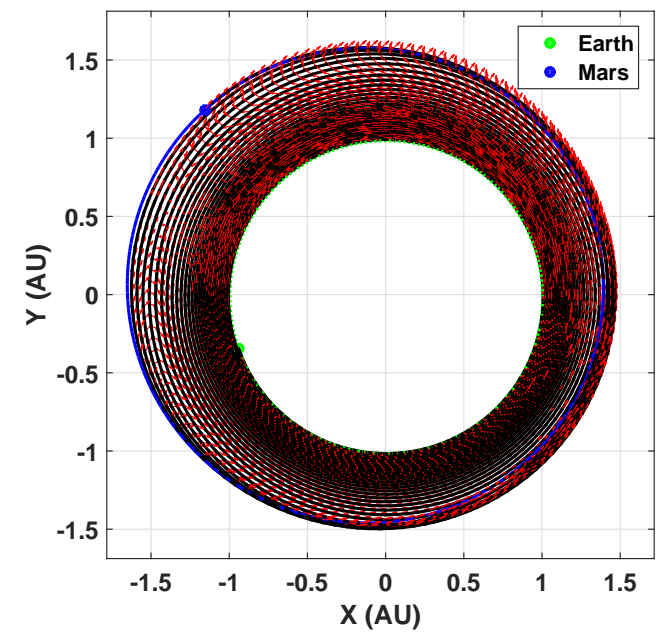

Figure 5: Minimum-time trajectory for the given BCs with $N_{\text {rev }}=30$

the GA. The computational time of the SB initialization which includes the time it takes for a SB method to find a feasible solution plus the solution of the least squares problem of Eq. (28) along with the computation time of the final problem is still less than a GA which utilizes multi cores. From Table 3 we note that, our method has higher rate of convergence with less computational time compared to GA that uses multiple cores.

\subsection{Earth-to-Dionysus problem}

In this section, we are evaluating the performance of our technique when solving a more difficult problem in which the differences between initial and final orbital elements are significantly large in comparison with the Earthto-Mars problem. The orbital elements of asteroid Dionysus are taken from ${ }^{1}$, see Table 4 in which the Epoch date is given as the Modified Julian Date (MJD).

The target body is asteroid Dionysus, the heliocentric orbit of which has eccentricity and inclination values of 0.542 and $13.54^{\circ}$, respectively, and makes many close approaches to Earth and this makes it an appealing destination for future missions. However, any low-thrust trajectory from Earth to

\footnotetext{
${ }^{1}$ Available online at http://ssd.jpl.nasa.gov (retrieved December 23, 2015)
} 
Table 4: Keplerian orbital elements of asteroid Dionysus wrt the Sun

\begin{tabular}{cccccccc}
\hline \hline & $a$ & $e$ & $i$ & $\Omega$ & $\omega$ & $M$ & Epoch \\
{$[\mathrm{AU}]$} & & {$[\mathrm{deg}]$} & $\begin{array}{c} \\
{[\mathrm{deg}]}\end{array}$ & $\begin{array}{c}{[\mathrm{deg}]} \\
{[\mathrm{deg}]}\end{array}$ & {$[\mathrm{MJD}]$} \\
\hline Dionysus & 2.2 & 0.542 & 13.6 & 82.2 & 204.2 & 114.4232 & 53400 \\
\hline
\end{tabular}

asteroid Dionysus requires considerable change in eccentricity and inclination values over several revolutions around Sun.

The BCs for the position and velocity of Earth and of asteroid Dionysus are

$$
\begin{aligned}
\mathbf{r}_{i} & =[-3637871.08,147099798.78,-2261.44]^{T}(\mathrm{~km}), \\
\mathbf{v}_{i} & =\left[-30.2650,-0.848685,5.053 \times 10^{-6}\right]^{T}(\mathrm{~km} / \mathrm{s}), \\
\mathbf{r}_{T} & =[-3.0245201488,3.1609717963,0.8287229007]^{T} \times 10^{8}(\mathrm{~km}) \\
\mathbf{v}_{T} & =[-4.5334737,-13.1103098,0.65616382]^{T}(\mathrm{~km} / \mathrm{s}) .
\end{aligned}
$$

Note that these BCs correspond to a mission with departure from Earth on December 23, 2012 that takes 3534 days to reach to the target body (Vasile et al., 2007). Similar to the Earth-to-Mars problem, the BCs are fixed and only the engine maximum thrust value is varied to result in different trajectories.

In the numerical simulations, the specific impulse is $I_{s p}=3000 \mathrm{~s}$ and the initial mass of the spacecraft is $m_{0}=4000 \mathrm{~kg}$. Initially, we set a maximum thrust value to $T_{\max }=0.16 \mathrm{~N}$ that corresponds to a moderate value for the number of revolutions i.e., $N_{\text {rev }}=5$ and use PE and 3D FFS SB methods for co-state initialization.

In the next step, we consider a lower value for the maximum thrust $T_{\max }=0.053 \mathrm{~N}$ which corresponds to $N_{\text {rev }}=15$ and repeat the solution procedure. Table 5 summarizes the convergence rate with different approaches for the cases in which $N_{\text {rev }}=15$. For the case with $N_{\text {rev }}=5$ the rate of convergence of our proposed method is $100 \%$. For $N_{\text {rev }}=15$, the convergence rate decreases but the difference between our proposed approach and those of the other approaches is still noticeable.

The numbers in Table 5 indicate that random initialization has the worst performance. Note that we have considered two variants of ACT i.e., when PE is used with the ACT (PE-ACT) and when 3D FFS is used with ACT (3D FFS-ACT). The performance of our proposed approach is still better than those of PE-ACT and 3D FFS-ACT. 
Table 5: Comparison between the convergence rate of the approaches for the given BCs for $N_{\text {rev }}=15$.

\begin{tabular}{|c|c|}
\hline Initialization Method & Rate of convergence \\
\hline PE & $58 \%$ \\
3D FFS & $56 \%$ \\
PE-ACT & $46 \%$ \\
3D FFS-ACT & $38 \%$ \\
GAFM (Popu. 5000, Gen. 5) & $34 \%$ \\
GAFM (Popu. 2000, Gen. 5) & $24 \%$ \\
Random & $12 \%$ \\
\hline
\end{tabular}

Figures 6 and 7 show the evolution of the radius for the initial PE, 3D FFS and the minimum-time solutions (labeled as 'PMP') for $N_{r e v}=5$ and $N_{\text {rev }}=$ 15 which corresponds to $T_{\max }=0.16 \mathrm{~N}$ and $T_{\max }=0.053 \mathrm{~N}$, respectively.

Note that the time of flight of the solution of PE and 3D FFS SB methods are smaller than the minimum-time solution. The reason is due to the fact that when we used SB methods we did not consider the constraint on the maximum value of the thrust acceleration which means that the propulsion system is considered to be more capable compared to its true capability. Note that the state-of-the-art SB methods are capable of satisfying thrust acceleration constraints rather than the thrust itself.

Note that for the case, $N_{\text {rev }}=5$, the evolution of the radius with the 3D FFS technique is closer to that of the optimal minimum-time solution. For $N_{\text {rev }}=5$, the considered numbers of Fourier terms are $n_{r}=6, n_{\theta}=6$ and $n_{z}=8$, respectively. However, for $N_{\text {rev }}=15$, the evolution of radius with the PE SB method is closer to that of the minimum-time solution. The poor performance with the 3D FFS SB co-state initialization for the cases with a higher number of revolutions is mainly attributed to the number of considered Fourier terms and the fact that the approach uses the cylindrical CS which may not be the best choice for many revolution problems. However, the convergence rate with the $3 \mathrm{D}$ FFS SB co-state initialization is $56 \%$ which is not significantly different compared to the convergence rate with the PE SB co-state initialization technique which is $58 \%$. For, $N_{\text {rev }}=15$, the considered numbers of Fourier terms are $n_{r}=16, n_{\theta}=16$ and $n_{z}=20$, respectively.

In Figure 7, there is a gradual shift in the peak-to-peak frequency between the solution of the PE SB method and that of the minimum-time solution. It can be attributed to 1) the fixed value of the TOF which is considered when using the PE SB method, 2) the exponential approximation of the pseudo-elements in the PE SB method. The complete trajectory of 
the pseudo-elements entails secular as well as periodic variations. However, in the PE SB method (Pascale and Vasile, 2006) the emphasis has been to come up with a simple approximation function that is capable of capturing the secular variation of pseudo-elements to avoid introducing too many design parameters. In addition, neither the solution of 3D FFS nor that of PE are optimal, in the minimum-time sense.

Note also that in the numerical simulations, the TOF of the solutions of $\mathrm{PE}$ and 3D FFS SB techniques are equal to each other and fixed. However, they have significantly improved the rate of convergence of the shooting problem of Eq. (22) in comparison to the random initialization.

Note that the performance of the PE-ACT and 3D FFS-ACT is also better than the hybrid GAFM technique. Table 6 summarizes the best minimumtime solutions that we found for each thrust value. Figures 8 and 9 show the trajectories for the minimum-time Earth-to-Dionysus problem when $N_{\text {rev }}=5$ and $N_{r e v}=15$, respectively. Note that there is no guarantee that the minimum-time solutions are global and they only satisfy the necessary conditions of optimality.

For instance, knowing a relatively good value for the TOF, we solve the single-shooting problem of Eq. (22) with an initial value for $T O F=11000$ days and 100 random initialization of co-states $\boldsymbol{\lambda}\left(t_{i}\right)$. The elements of the initial co-state vector belong to the interval $[0,1]$. From numerous experiments, the minimum-time solution that we found is of 10993.58 days, which is 95.52 days shorter than the solution that we found through our co-state estimation approach, and corresponds to $0.86 \%$ difference. We should emphasize that the knowledge of the time of flight has been used to obtain the former better solution.

Table 6: Minimum time of flight for each thrust levels for the given BCs.

\begin{tabular}{|c|c|c|}
\hline$T_{\max }(\mathrm{N})$ & 0.16 & 0.053 \\
\hline$N_{\text {rev }}$ & 5 & 15 \\
\hline TOF (days) & 3693.25 & 11089.1 \\
\hline
\end{tabular}

We further test the performance of the GA when different combinations of population and generation numbers are considered. Figure 10 compares the rate of convergence for our proposed methods versus that of GA+fmincon.

The surface presents the rate of convergence, i.e., the number of times that a feasible solution was found in 100 simulations for GA+fmincon. Each simulation corresponds to running GA and using the co-states of the solution 


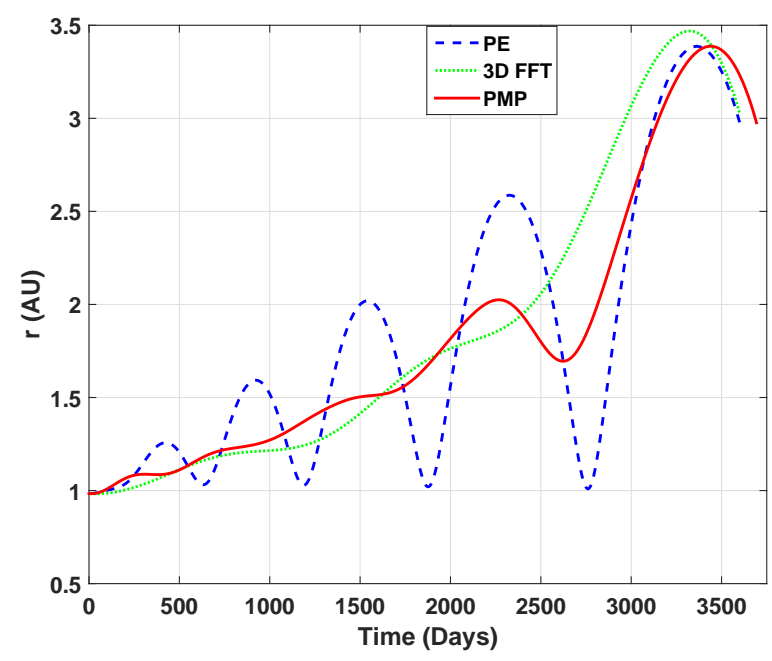

Figure 6: Evolution of radius for the given BCs with $N_{\text {rev }}=5$

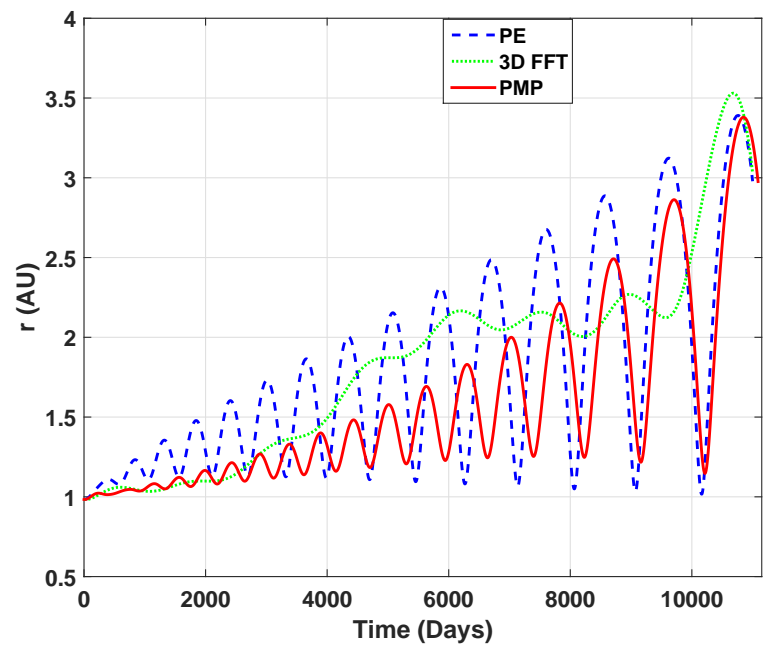

Figure 7: Evolution of radius for the given BCs with $N_{\text {rev }}=15$ 


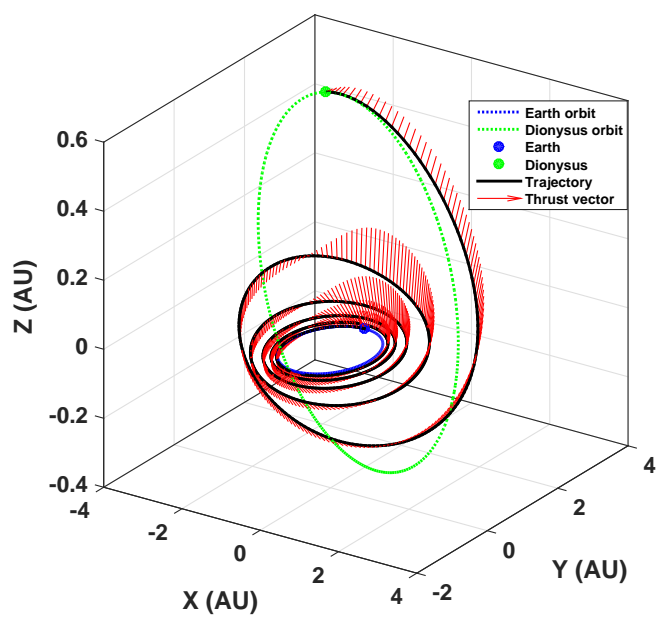

Figure 8: Minimum-time trajectory for the given BCs with $N_{\text {rev }}=5$

of GA as an initial guess for fmincon. We can see that as the number of generations and size of populations increase, the performance of the GA with respect to the percent of converged cases improves; but still the performance of our proposed PE and FFS costates initialization method surpasses the results by GA even when GA uses 10 generations and 5000 population size, which are the maximum numbers in our comparison. And the PE+ACT and FFS+ACT methods demonstrate better or comparable performance versus GA.

Figure 11 shows the average required computational time by different methods. As the number of generations and size of populations of GA increase, the computational time increases exponentially.

Compared to GA, the time required by our proposed algorithm is significantly lower considering that GA utilizes parallel computing capability of MATLAB with four cores whereas the other methods run on a single core. As the results show, our proposed method surpasses GA both in terms of percent of converged cases as well as the computational time.

Note that both of the solvers used in this paper are MATLAB functions and are not inherently fast. We have already used a compiled propagator for all of the methods, and trajectory propagation is the most computationally intensive part of the problem. All of the methods will be faster if coded in a compiled programming language like $\mathrm{C}$ or Fortran. However, the ratio 


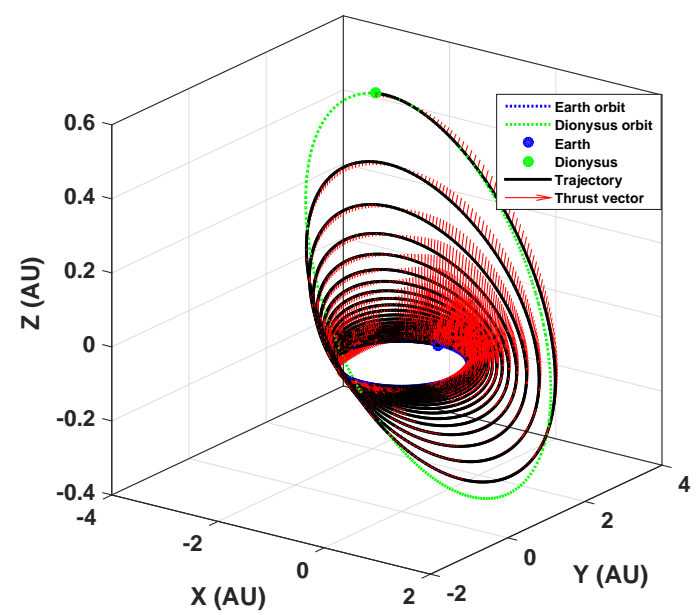

Figure 9: Minimum-time trajectory for the given BCs with $N_{\text {rev }}=15$

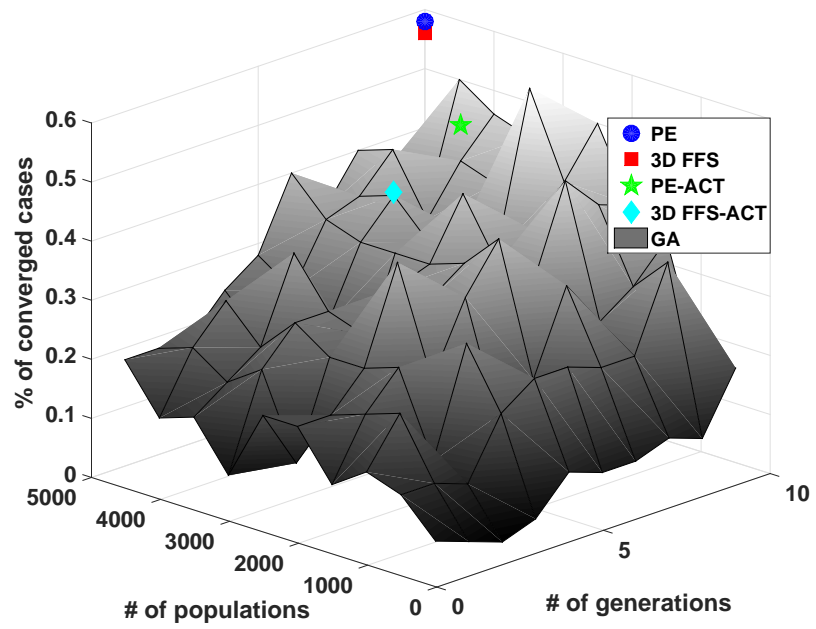

Figure 10: Comparison of the percent of converged cases for GA with different numbers of populations and generations versus the other methods.

between the required simulation time is not affected. The algorithm speed is a key factor for methods that are developed for broad trajectory search in the preliminary design. As the results demonstrate, our method has desirable 


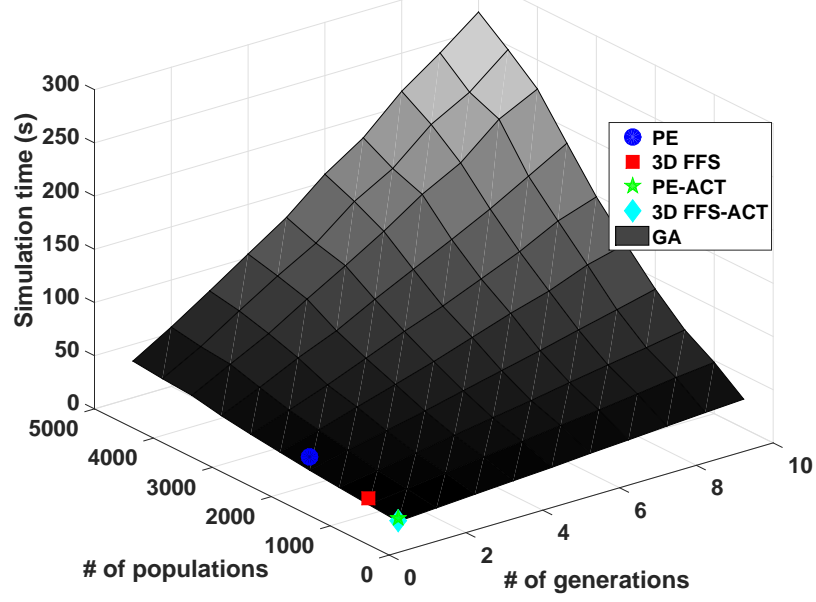

Figure 11: Comparison of simulation time for GA (running in parallel using four cores) versus the other methods.

characteristics with high percent of convergence and low computational time and effort versus alternatives that we have considered. Note that if the analytic gradient is available, those solvers that are based on Newton-update algorithms will have much better convergence and computational efficiency than the usual finite-difference method. Calculation of the analytic gradients and their utilization belongs to the future works.

Note that the final solution obtained by the proposed method depends, to some extent, on the original SB solution, from which the initial values of the co-states are reconstructed. For instance, when we use either of the SB methods to generate a feasible trajectory, the number of revolutions is prespecified. As a result, the solutions to the TPBVP found through our co-state estimation approach mostly match this pre-specified number of revolutions. This may lead to local optimality of the converged solution. 


\section{CONCLUSIONS}

This paper proposed a trajectory optimization approach that exploits the shape-based method to estimate the initial co-states. The proposed approach was applied successfully to two interplanetary minimum-time rendezvous problems from Earth to Mars and from Earth to asteroid Dionysus. The latter problem is a difficult problem for any direct optimal control solver to handle due to the greater change in the eccentricity and inclination over a long time of flight with several number of revolutions around the Sun. For each problem, various levels of thrust and number of revolutions were considered.

We have used and compared two shape-based methods that are capable

of generating three-dimensional trajectories, i.e., the pseudo-equinoctial and three-dimensional finite Fourier series shape-based methods.

The performance of the proposed approach was compared against the random initialization of co-states, a standard genetic algorithm and the adjointcontrol transformation method. We showed that in terms of the convergence rate and computational demand, the proposed approach can outperform other techniques especially when the number of revolutions is high. In particular, the random initialization of the values of the co-states results in poor convergence performance.

Combining the shape-based method with the adjoint-control transformation has also been studied. The results indicate that the convergence rate with this combined approach was improved compared to with a pure random initialization and was also better than with a standard genetic algorithm. However, it is still inferior to our proposed method which exploits the solution of the shape-based methods to estimate the initial value of the adjoint variables. On the other hand, the performance of the method depends on the quality of the shape-based solution. For the cases with many revolutions, the number of revolutions of the solution obtained from the proposed method tends to match the pre-specified value of the shape-based solution.

However, despite the fact that the estimate values of the co-states are not necessarily very close to those of the optimal solutions, they do improve the convergence rate. The analysis of the initial estimates of the co-states suggests that there exist a relationship between the co-states that has a far more important impact on improving the convergence performance.

The relationship between the values of the co-states and the extension of the method to the restricted three-body dynamics belong to the future 
works.

\section{Acknowledgments}

The authors would like to acknowledge the support of the National Science Foundation under the Award Number CNS 1544844.

\section{References}

Ossama Abdelkhalik and Ahmed Gad. Dynamic-size multiple populations genetic algorithm for multigravity-assist trajectory optimization. Journal of Guidance, Control, and Dynamics, 35(2):520-529, doi:10.2514/1.54330, 2012.

Régis Bertrand and Richard Epenoy. New smoothing techniques for solving bang-bang optimal control problems - numerical results and statistical interpretation. Optimal Control Applications and Methods, 23(4):171-197, doi:10.1002/oca.709, 2002.

John T. Betts. Survey of numerical methods for trajectory optimization. Journal of Guidance, Control, and Dynamics, 21:93-207, doi:10.2514/2.4231, 1998.

Christian M Chilan and Bruce A Conway. Automated design of multiphase space missions using hybrid optimal control. Journal of Guidance, Control, and Dynamics, 36(5):1410-1424, doi:10.2514/1.58766, 2013.

LCW Dixon and Michael C Bartholomew-Biggs. Adjoint-control transformations for solving practical optimal control problems. Optimal Control Applications and Methods, 2(4):365-381, doi:10.1002/oca.4660020405, 1981.

Jacob A. Englander, Bruce A. Conway, and Trevor Williams. Automated mission planning via evolutionary algorithms. Journal of Guidance, Control, and Dynamics, 35(6):1878-1887, doi:10.2514/1.54101, 2012.

J Fourcade, S Geffroy, and R Epenoy. An averaging optimal control tool for low-thrust minimum-time transfers. CNES, editor, Low thurst trajectory optimization, 2000. 
Sophie Geffroy and Richard Epenoy. Optimal low-thrust transfers with constraints - generalization of averaging techniques. Acta Astronautica, 41(3):133-149, 1997.

David E. Goldberg. Genetic Algorithms in Search, Optimization and Machine Learning. Addison-Wesley Longman Publishing Co., Inc., Boston, MA, USA, 1st edition, 1989. ISBN 0201157675.

David J. Gondelach and Ron Noomen. Hodographic-shaping method for lowthrust interplanetary trajectory design. Journal of Spacecraft and Rockets, 52(3):728-738, doi:10.2514/1.A32991, 2015.

Daniel Hennes and Dario Izzo. Interplanetary trajectory planning with monte carlo tree search. In Proceedings of the 24th International Conference on Artificial Intelligence, AAAI Press, pages 769-775, 2015.

Fanghua Jiang, Hexi Baoyin, and Junfeng Li. Practical techniques for lowthrust trajectory optimization with homotopic approach. Journal of guidance, control, and dynamics, 35(1):245-258, 2012.

Reza Kamyar and Ehsan Taheri. Aircraft optimal terrain/threat-based trajectory planning and control. Journal of Guidance, Control, and Dynamics, 37(2):466-483, doi:10.2514/1.61339, 2014.

Craig A. Kluever and Bion L. Pierson. Optimal earth-moon trajectories using nuclear electric propulsion. Journal of Guidance, Control, and Dynamics, 20(2):239-245, doi:10.2514/2.4058, 1997.

Gregory Lantoine and Ryan P. Russell. A hybrid differential dynamic programming algorithm for constrained optimal control problems. part 2: Application. Journal of Optimization Theory and Applications, 154(2):418442, doi:10.1007/s10957-012-0038-1, 2012.

Daniel M. Novak and Massimiliano Vasile. Improved shaping approach to the preliminary design of low-thrust trajectories. Journal of Guidance, Control, and Dynamics, 34(1):128-147, doi:10.2514/1.50434, 2011.

Paolo De Pascale and Massimiliano Vasile. Preliminary design of low-thrust multiple gravity assist trajectories. Journal of Spacecraft and Rockets, 43: 1065-1076, doi:10.2514/1.19646, 2006. 
Frank M. Perkins. Flight mechanics of low-thrust spacecraft. Journal of the Aerospace Sciences, 26(5):291-297, 1959.

Chris L. Ranieri and Cesar A. Ocampo. Optimization of roundtrip, timeconstrained, finite burn trajectories via an indirect method. Journal of Guidance, Control, and Dynamics, 28(2):306-314, doi:10.2514/1.5540, 2005 .

Ryan P. Russell. Primer vector theory applied to global low-thrust trade studies. Journal of Guidance, Control, and Dynamics, 30(2):460-472, 2007.

Juan Senent, Cesar Ocampo, and Antonio Capella. Low-thrust variablespecific-impulse transfers and guidance to unstable periodic orbits. Journal of Guidance, Control, and Dynamics, 28(2):280-290, doi:10.2514/1.6398, 2005 .

Ehsan Taheri and Ossama Abdelkhalik. Approximate on-off low-thrust space trajectories using fourier series. Journal of Spacecraft and Rockets, 49(5): 962-965, doi:10.2514/1.A32307, 2012a.

Ehsan Taheri and Ossama Abdelkhalik. Shape based approximation of constrained low-thrust space trajectories using fourier series. Journal of Spacecraft and Rockets, 49(3):535-546, doi:10.2514/1.A32099, 2012b.

Ehsan Taheri and Ossama Abdelkhalik. Fast initial trajectory design for low-thrust restricted-three-body problems. Journal of Guidance, Control, and Dynamics, 38(11):2146-2160, doi:10.2514/1.G000878, 2015.

Ehsan Taheri and Ossama Abdelkhalik. Initial three-dimensional low-thrust trajectory design. Advances in Space Research, 57(3):889-903, 2016.

Ehsan Taheri, Ilya Kolmanovsky, and Ella Atkins. Enhanced smoothing technique for indirect optimization of minimum-fuel low-thrust trajectories. Journal of Guidance, Control, and Dynamics, 39(11):2500-2511, 2016a.

Ehsan Taheri, Nan I. Li, and Ilya Kolmanovsky. Co-states initialization of minimum-time low-thrust trajectories using shape-based methods. In Proceedings of the 2016 American Control Conference, pages 4053-4058. AIAA, 2016b. 
James D. Thorne and Christopher D. Hall. Approximate initial lagrange costates for continuous-thrust spacecraft. Journal of guidance, control, and dynamics, 19(2):283-288, doi:10.2514/3.21616, 1996.

Massimiliano Vasile, Paolo De Pascale, and Stefano Casotto. On the optimality of a shape-based approach based on pseudo-equinoctial elements. Acta Astronautica, 61(1):286-297, doi:10.1016/j.actaastro.2007.01.017, 2007.

Massimiliano Vasile, Edmondo Minisci, and Marco Locatelli. Analysis of some global optimization algorithms for space trajectory design. Journal of Spacecraft and Rockets, 47(2):334-344, doi:10.2514/1.45742, 2010.

Bradley J. Wall and Bruce A. Conway. Shape-based approach to low-thrust rendezvous trajectory design. Journal of Guidance, Control, and Dynamics, 32(1):95-101, doi:10.2514/1.36848, 2009.

Hui Yan and Hongxin Wu. Initial adjoint-variable guess technique and its application in optimal orbital transfer. Journal of guidance, control, and dynamics, 22(3):490-492, doi:10.2514/2.7631, 1999.

Xincheng Yue, Ying Yang, and Zhiyong Geng. Continuous low-thrust timeoptimal orbital maneuver. In Decision and Control, 2009 held jointly with the 2009 28th Chinese Control Conference. CDC/CCC 2009. Proceedings of the 48th IEEE Conference on, pages 1457-1462. IEEE, 2009. 